\title{
APOGEE Chemical Abundances of Globular Cluster Giants in the Inner Galaxy
}

Ricardo P. Schiavon ${ }^{1 \star}$, Jennifer A. Johnson ${ }^{2}$, Peter M. Frinchaboy ${ }^{3}$, Gail Zasowski ${ }^{4}$, Szabolcs Mészáros ${ }^{5}$, D. A. García-Hernández ${ }^{6,7}$, Roger E. Cohen ${ }^{8}$, Baitian Tang 8 , Sandro Villanova ${ }^{8}$, Douglas Geisler ${ }^{8}$, Timothy C. Beers ${ }^{9}$, J. G. Fernández-Trincado ${ }^{10}$, Ana E. García Pérez ${ }^{11}$, Sara Lucatello ${ }^{12}$, 'Steven R. Majewski ${ }^{11}$, Sarah L. Martell ${ }^{13}$, Robert W. O'Connell ${ }^{11}$, Carlos Allende Prieto ${ }^{6,7}$, Dmitry Bizyaev ${ }^{14,15}$, Ricardo Carrera ${ }^{6,7}$, Richard R. Lane ${ }^{16}$, Elena Malanushenko ${ }^{14}$, Viktor Malanushenko ${ }^{14}$, Ricardo R. Muñoz ${ }^{17}$, Christian Nitschelm ${ }^{18}$, Daniel Oravetz ${ }^{14}$, Kaike Pan $^{14}$, Alexandre Roman-Lopes ${ }^{19}$, Matthias Schultheis ${ }^{20} \&$ Audrey Simmons ${ }^{14}$.

${ }^{1}$ Astrophysics Research Institute, Liverpool John Moores University, 146 Brownlow Hill, Liverpool, L3 5RF, United Kingdom

${ }^{2}$ Department of Astronomy, The Ohio State University, Columbus, OH 43210, USA

${ }^{3}$ Texas Christian University, Fort Worth, TX 76129, USA

${ }^{4}$ Department of Physics and Astronomy, Johns Hopkins University, Baltimore, MD 21218, USA

${ }^{5}$ ELTE Gothard Astrophysical Observatory, H-9704 Szombathely, Szent Imre Herceg st. 112, Hungary

${ }^{6}$ Instituto de Astrofísica de Canarias, E-38205 La Laguna, Tenerife, Spain

${ }^{7}$ Departamento de Astrofísica, Universidad de La Laguna (ULL), E-38206 La Laguna, Tenerife, Spain

${ }^{8}$ Departamento de Astronomía, Casilla 160-C, Universidad de Concepción, Concepción, Chile

${ }^{9}$ Dept. of Physics and JINA Center for the Origin of the Elements, University of Notre Dame Notre Dame, IN 46530 USA

${ }^{10}$ Institut Utinam, CNRS UMR6213, Université de Franche-Comté, OSU THETA Franche-Comté-Bourgogne, Observatoire de Besançon, BP 1615, 25010 Besançon Cedex, France

${ }^{11}$ Dept. of Astronomy, University of Virginia, Charlottesville, VA 22904-4325, USA

${ }^{12}$ INAF-Osservatorio Astronomico di Padova, Vicolo dellOsservatorio 5, I-35122 Padova, Italy

${ }^{13}$ School of Physics, University of New South Wales, Sydney, NSW 2052, Australia

${ }^{14}$ Apache Point Observatory and New Mexico State University, P.O. Box 59,

Sunspot, NM 88349-0059, USA

${ }^{15}$ Sternberg Astronomical Institute, Moscow State University, Moscow

${ }^{16}$ Instituto de Astrofísica, Pontificia Universidad Católica de Chile, Av. Vicuna Mackenna 4860, 782-0436 Macul, Santiago, Chile

17 Departamento de Astronomía, Universidad de Chile, Camino El Observatorio 1515, Las Condes, Santiago, Chile

${ }^{18}$ Unidad de Astronomía, Universidad de Antofagasta, Avenida Angamos 601, Antofagasta 1270300, Chile

${ }^{19}$ Departamento de Fúsica, Facultad de Ciencias, Universidad de La Serena, Cisternas 1200, La Serena, Chile

${ }^{20}$ Laboratoire Lagrange (UMR7293), Universite de Nice Sophia Antipolis, CNRS, Observatoire de la C ote dAzur, BP 4229, F-06304 Nice Cedex 4, France

Draft, 4 November, 2016

\begin{abstract}
We report chemical abundances obtained by SDSS-III/APOGEE for giant stars in five globular clusters located within $2.2 \mathrm{kpc}$ of the Galactic centre. We detect the presence of multiple stellar populations in four of those clusters (NGC 6553, NGC 6528, Terzan 5, and Palomar 6) and find strong evidence for their presence in NGC 6522. All clusters present a significant spread in the abundances of N, C, Na, and Al, with the usual correlations and anti-correlations between various abundances seen in other globular clusters. Our results provide important quantitative constraints on theoretical models for self-enrichment of globular clusters, by testing their predictions for the dependence of yields of elements such as $\mathrm{Na}, \mathrm{N}, \mathrm{C}$, and $\mathrm{Al}$ on metallicity. They also confirm that, under the assumption that field N-rich stars originate from globular cluster destruction, they can be used as tracers of their parental systems in the highmetallicity regime.
\end{abstract}

\section{Key words:}




\section{INTRODUCTION}

The discovery, within the past decade, of the presence of multiple stellar populations in Galactic globular clusters (GCs) has forced a revision of the traditional paradigm for the origin of these objects. For several decades, GC stars have been known to exhibit $\mathrm{Na}, \mathrm{O}, \mathrm{Al}$, and $\mathrm{Mg}$ (e.g., Gratton et al. 2004; Carretta et al. 2010) spreads. More recently, star-to-star variations in chemical-composition were associated with the detection of multiple sequences in highprecision colour-magnitude diagrams for the majority of Galactic GCs (e.g., Piotto 2008), leading to the suggestion of a complex history of star formation and chemical enrichment in systems once thought to be prototypical single stellar populations. Under most such scenarios, the so-called "firstgeneration" (FG) stars exhibit abundance patterns that are similar to those of field stars of the same $[\mathrm{Fe} / \mathrm{H}]$, whereas the chemical compositions of "second-generation" (SG) stars depart from those patterns, showing enhancement in light elements such as $\mathrm{He}, \mathrm{N}, \mathrm{Na}$, and $\mathrm{Al}$, and depletion in $\mathrm{C}, \mathrm{O}$, and sometimes $\mathrm{Mg}$.

No theoretical models based on the premise that GCs evolve chemically have thus far been able to account for the extant data in detail (see Renzini et al. 2015, for a review), so other alternatives have been sought (e.g., Bastian et al. 2013; Hopkins 2014), and those have also been shown to fail (Bastian et al. 2015). Naively, self-enrichment models seem reasonable, for they frame GCs as low-mass manifestations of processes of star formation and chemical enrichment known to operate in galaxies - a notion that is particularly supported by the detection of mass-chemical composition relations in GCs (Carretta et al. 2010; Schiavon et al. 2013; Sakari et al. 2016). However, to be tenable they must rely on requirements that are not borne out by the data. Chief amongst those is the so called "mass budget problem", according to which, for any assumed initial mass function, the observed mass of freshly produced nucleosynthetic material currently observed in the atmospheres of SG stars requires a much larger number of FG polluters than can be reconciled with the numbers of existing low-mass FG cluster stars (e.g., Renzini 2008) - typically by one or more orders of magnitude.

This assumption has been recently challenged (Schiavon et al. 2016) by the discovery of a large population of stars with enhanced $\mathrm{N}$ abundances (N-rich stars) in the field of the inner Galaxy. The abundance patterns of these stars resemble those of SG stars in Galactic GCs, suggesting that they are the possible leftovers of a large population of early GCs that were entirely destroyed. Interestingly, the maximum ratio between FG and SG stars in the inner Galaxy is lower than required by models to solve the mass budget problem (see also Larsen et al. 2014). Moreover, the lower limit to the mass contained in stars originated in the presumptive destroyed GCs is larger than that of the existing Galactic GC system by an order of magnitude. Since the metallicity distribution function of N-rich stars is substantially different from that of the present GC system (Harris 1996), one is led to conclude that the remaining GCs are not simply a scaled-down version of a much larger precursor GC system. Rather, the mass loss by the existing GCs was probably modest, and the GC-like stars found in the field today come from a parental GC population that was mostly destroyed. The latter conclusion, though potentially far reaching, depends crucially on the assumption that N-rich stars are reliable tracers of GC populations at all metallicities - in other words, that SG stars in metal-rich GCs present the same levels of enrichment/depletion in light elements as their more metal-poor counterparts.

Progress in this field depends crucially on the mapping of the multiple-population phenomenon across the entire volume of parameter space covered by GCs, with metallicity being a particularly important parameter. Painstaking observational efforts have yielded a large collection of colour-magnitude diagrams (e.g., Piotto et al. 2002, 2015) and detailed abundance patterns of GC members (e.g., Carretta et al. 2010; Pancino et al. 2010; Mészáros et al. 2015). On the metal-rich end, however, elemental abundances are not available for large samples of GC members, because most metal-rich GCs are located in the inner Galaxy, which is difficult to access in the optical due to large dust extinction. To our knowledge, only a very small number of metal-rich GCs have been studied with sufficiently large member samples to enable the detection of multiple stellar populations, although low-resolution spectroscopy of stellar members (e.g., Martell \& Smith 2009; Pancino et al. 2010) and integrated spectroscopy of extragalactic GCs (Schiavon et al. 2013) suggests that they are present.

It is important to document the presence of multiple populations in metal-rich GCs for additional reasons. Firstly, multiple populations provide critical tests of stellarevolution model predictions for yields of light elements (e.g. Karakas 2010; Ventura et al. 2013; Di Criscienzo et al. 2016) in a regime that is important not only for the debate over globular cluster formation, but also in the context of models for the chemical evolution of galaxies (e.g., Pipino et al. 2009). Secondly, an estimate of the total mass contained in the presumptive dissolved GCs discovered by Schiavon et al. (2016) in the inner Galaxy depends on knowledge of the ratios between FG and SG stars across the entire metallicity range. Thirdly, there is evidence for the existence of a positive correlation between the amplitude of abundance spreads in GCs and their masses and metallicities, resulting from analysis of the abundances of $\mathrm{Na}$ and $\mathrm{O}$ in individual Galactic GC stars (Carretta et al. 2010) and, in an indirect way, from the mean $\mathrm{N}$ abundances of M31 GCs (Schiavon et al.2013). One would thus expect to find similar abundance spreads in metal-rich Galactic GCs of moderateto-high masses.

High-resolution near-infrared (NIR) spectroscopy for large samples of stars is probably the most efficient way to attack this problem. The Apache Point Observatory Galactic Evolution Experiment (APOGEE, Majewski et al. 2016) contributes importantly in that regard. A massive survey of Galactic stellar populations, APOGEE obtained H-band $R \sim 22,500$ resolution spectra for over 150,000 stars, many 
of which are members of globular clusters. This article reports the abundance patterns of 23 candidate members of five GCs situated within the inner Galaxy. Sample and data are described in Section 2 and results are presented in Section 3. Our conclusions are summarised in Section 4 A more exhaustive evaluation of membership and an analysis of the detailed abundance patterns of members of one of the GCs in our sample (NGC 6553) are presented in a separate paper (Tang et al. 2016). For a detailed analysis of APOGEE abundances for members of GCs outside the inner Galaxy, we refer the reader to Mészáros et al. (2015).

\section{DATA AND SAMPLE}

\subsection{Target Selection}

The rationale behind targeting GC stars in APOGEE was twofold: on the one hand, stars that were previously subjected to detailed abundance analysis are useful for reality checks and potentially the calibration of APOGEE elemental abundances. On the other hand, there is an obvious interest in expanding the database of GC elemental abundances by targeting stars that are known or probable GC members, but whose chemical compositions are unknown. The range of metallicities of our sample GCs overlaps largely with samples from previous APOGEE studies (Mészáros et al. 2015; Schiavon et al. 2016), but does include some of the most metal-rich GCs known in the Galaxy (NGC 6528 and 6553). Also targeted is a GC known to host at least three populations with distinct $[\mathrm{Fe} / \mathrm{H}]$ (Terzan 5), and two more metalpoor GCs (NGC 6522 and Palomar 6). The main relevant properties of the target GCs are summarised in Table 2.1 For each GC, lists of candidate members were put together including targets from both categories above, and these lists were fed to a prioritization algorithm that assigned fibers to various targets in each APOGEE field. Fiber collision poses a major limitation against a dense sampling of GC stars, by preventing the simultaneous observation of targets separated by less than $1^{\prime}$. However, more stars could be observed from GCs located in fields that were visited multiple times. As a result of these constraints, the sampling of the target GCs discussed in this paper is somewhat serendipitous and not evenly distributed, ranging between one candidate member for NGC 6522 and twelve for NGC 6553. All targets are giant stars that are likely GC members with stellar parameters within the following range: $3600 \lesssim T_{\text {eff }} \lesssim 4700 \mathrm{~K}$ and $0 \lesssim \log g \lesssim 2.6$. For further details on APOGEE target selection, we refer the reader to Zasowski et al. (2013).

\section{$2.2 \quad$ Data}

The results presented in this paper are based on the products of Data Release 12 (DR12, Alam et al. 2015; Holtzman et al. 2015) of the SDSS-III/APOGEE survey (Eisenstein et al. 2011; Majewski et al. 2016), consisting of accurate elemental abundances and radial velocities, supplemented here by 2MASS astrometry (Skrutskie et al. 2006) and GC structural parameters and radial velocities from the 2010 edition of the Harris catalog of Galactic globular clusters (Harris 1996, 2010). Elemental abundances are based on the automatic analysis of APOGEE spectra performed by the APOGEE Stellar Parameter and Chemical Abundances Pipeline (ASPCAP, García Pérez et al. 2016), which performs a quantitative comparison of observed spectra with a huge spectral library, calculated on the basis of state-of-the-art model atmospheres (Mészáros et al. 2012; Zamora et al. 2015) and a comprehensive and accurate line list (Shetrone et al. 2015). The stellar spectra were themselves collected with the APOGEE spectrograph (Wilson et al. 2012; Majewski et al. 2016) attached to the Sloan 2.5m telescope (Gunn et al. 2006) at Apache Point Observatory. A detailed description of the data reduction and resulting data products can be found in Nidever et al. (2015) and Holtzman et al. (2015).

We focus on $[\mathrm{Fe} / \mathrm{H}]$ and abundance ratios whose star to star variations within GCs are the typical indicators of the presence of multiple stellar populations, such as $[\mathrm{C} / \mathrm{Fe}]$, $[\mathrm{N} / \mathrm{Fe}],[\mathrm{Na} / \mathrm{Fe}],[\mathrm{Al} / \mathrm{Fe}]$, and $[\mathrm{Mg} / \mathrm{Fe}]$. While oxygen tends to be low in SG stars, ASPCAP currently does not provide reliable oxygen abundances for O-poor stars, so we leave that element out of the analysis in this paper. The typical precision of APOGEE abundances is better than $0.1 \mathrm{dex}$, which is more than adequate for our present purposes. The precision of APOGEE radial-velocities is typically better than $\sim 0.5 \mathrm{~km} / \mathrm{s}$, which again exceeds the requirements of our project.

\section{$2.3 \quad$ Membership}

Once stellar parameters and radial velocities of sample stars are known, their membership status was further scrutinised by filtering out all stars failing to meet projected distance, radial velocity, and metallicity criteria. In this way, stars with projected distances from GC centres that are larger than the GC tidal radii were removed from consideration. By the same token, stars with heliocentric radial velocities differing from the catalogued GC values by more than the GC velocity dispersion (or $\pm 15 \mathrm{~km} / \mathrm{s}$ in cases where velocity disperison is not available in the Harris catalog), and those differing in $[\mathrm{Fe} / \mathrm{H}]$ from the mean $\mathrm{GC}$ values by more than 0.3 dex were also removed from consideration. The GC centres, tidal radii, and metallicities adopted in these comparisons were taken from the 2010 version of the Harris (1996) catalog. The only exception is Terzan 5 , for which there is a large spread in $[\mathrm{Fe} / \mathrm{H}]$, so that no metallicity criterion was adopted. There is no evidence for the presence of $[\mathrm{Fe} / \mathrm{H}]$ variations in the other sample $\mathrm{GCs}$, so the $[\mathrm{Fe} / \mathrm{H}]$ criterion adopted was generous enough that no member stars are expected to be excluded from analysis. The final list of GC targets is listed in Table 2.3 together with elemental abundances, radial velocities, and distances from the host GC centres.

To estimate how many and which stars in the APOGEE sample were excluded due to adoption of the above selection criteria, we searched for candidate members meeting relaxed criteria by doubling the projected distance and radial veloc- 
Table 1. Properties of the globular clusters targeted in this study. Mean radial velocities and tidal radii are taken from the 2010 edition of the Harris (1996) catalogue. For references on $[\mathrm{Fe} / \mathrm{H}]$ and mass see text.

\begin{tabular}{ccrrr}
\hline \hline ID & {$[\mathrm{Fe} / \mathrm{H}]$} & $\langle\mathrm{RV}\rangle(\mathrm{km} / \mathrm{s})$ & $r_{t}(\operatorname{arcmin})$ & Mass $\left(\mathrm{M}_{\odot}\right)$ \\
\hline \hline Palomar 6 & -0.91 & +181.0 & 8.3 & $2.3 \times 10^{5}$ \\
Terzan 5 & {$[-1.2,+0.3]$} & -82.0 & 6.7 & $2.0 \times 10^{6}$ \\
NGC 6522 & -1.00 & -21.1 & 15.8 & $6.0 \times 10^{4}$ \\
NGC 6528 & -0.2 & +206.6 & 4.1 & $2.0 \times 10^{5}$ \\
NGC 6553 & -0.2 & -3.2 & 7.7 & $3.0 \times 10^{5}$ \\
\hline
\end{tabular}

ity search ranges. As a result, we found that adoption of these more relaxed criteria would have resulted in addition of a number of stars to our samples for NGC 6553, 6528, and Terzan 5. In most cases the additional stars fall within the range of metallicities acceptable for these three GCs, suggesting possible membership. However, all the stars in Table 2.3 have abundance patterns consistent with that of field samples at the same metallicity, which somewhat reduces the chances that they are associated with the GCs in our sample. We nevertheless list these additional stars in Table 2.3 for completeness, although they are not considered in our discussion.

\section{RESULTS}

The key result presented in this article is summarised in Figure 11 where data for all the members of the program GCs are displayed in various abundance-ratio planes. Symbol/colour codes are adopted to distinguish data for stars from different GCs, and on each panel the mean error bars are displayed. Overall, the stars from different GCs follow consistent trends in each diagram, exhibiting a clear C-N anti-correlation and also clear Na-N and Al-N correlations. As commonly seen in other samples (e.g., Mészáros et al. 2015), there is no clear anti-correlation between $\mathrm{Al}$ and $\mathrm{Mg}$ abundances, but rather a substantial spread in the abundance of the former and a smaller spread in $\mathrm{Mg}$ abundances. A bimodality is clearly seen in $[\mathrm{N} / \mathrm{Fe}]$ and $[\mathrm{C} / \mathrm{Fe}]$ but it is not present in other elemental abundances. The N-C bimodality is mostly driven by the data for NGC 6553 for which our sample is largest. There is a clear spread in the abundances of $\mathrm{Al}, \mathrm{Na}$, and $\mathrm{Mg}$, but no clear sign of a bimodal distribution can be distinguished for these elements. To decide whether this difference in behaviour between different elemental abundances is due to sample size, larger errors in the abundances of the latter elements, or a real physical effect, a larger sample will be required.

It is instructive to contrast the data for inner Galaxy GCs with those for GCs in the outer Galaxy. The grey circles in Figure 1 indicate the DR12 data for the halo/thickdisk GCs used by Schiavon et al. (2016), which are on average more metal-poor than the sample discussed in this paper. The GCs represented by grey symbols are M 3 $([\mathrm{Fe} / \mathrm{H}]=-1.5), \mathrm{M} 5([\mathrm{Fe} / \mathrm{H}]=-1.3), \mathrm{M} 107([\mathrm{Fe} / \mathrm{H}]=-1.0)$, M $71([\mathrm{Fe} / \mathrm{H}]=-0.8)$, and NGC $6760([\mathrm{Fe} / \mathrm{H}]=-0.4)$. Overall, the two sets of GCs occupy the same loci in the var- ious chemical-composition planes which is reassuring. But some differences are noteworthy. In the $\mathrm{C}-\mathrm{N}$ plane, there is a large collection of metal-poor $\mathrm{GCs}$ at $[\mathrm{C} / \mathrm{Fe}]<-0.2$ and $[\mathrm{N} / \mathrm{Fe}] \lesssim+0.5$. This is due to the presence of a large population of FG stars from M 3 and M 5, which have lower $[\mathrm{C} / \mathrm{Fe}]$ and slightly higher $[\mathrm{N} / \mathrm{Fe}]$ than their counterparts in more metal-rich GCs. This difference is likely to be the result of the evolution of $\mathrm{C}$ and $\mathrm{N}$ abundances in the Galaxy, a topic that is beyond the scope of this paper. Regarding $[\mathrm{Al} / \mathrm{Fe}]$, there is a hint that the more metal-poor GCs have a larger spread than their metal-rich counterparts, but that difference is barely significant given the size of the errors. The metal-poor GCs show a possible indication of the presence of a $\mathrm{Mg}-\mathrm{Al}$ anti-correlation, as there is a cluster of data with nearly solar $[\mathrm{Mg} / \mathrm{Fe}]$ and $[\mathrm{Al} / \mathrm{Fe}] \sim+0.6$. Those stars all belong to M 3, so they are fairly metal-poor. Interestingly, one of the Terzan 5 stars in our sample inhabits the same region of the $\mathrm{Mg}-\mathrm{Al}$ plane. With $[\mathrm{Fe} / \mathrm{H}]=-0.48$, this star is ten times more metal-rich than M 3 members, suggesting that the same process leading to the $\mathrm{Mg}-\mathrm{Al}$ anti-correlation may be present at high metallicity. Finally, we note that data for $[\mathrm{Na} / \mathrm{Fe}]$ are not shown for the metal-poor GCs, since that elemental abundance is quite uncertain in APOGEE DR12 for metal-poor GCs.

The abundances of some elements, such as nitrogen and carbon, are known to vary during evolution along the giant branch, both due to the first dredge-up and extra mixing further up that evolutionary sequence. It is important to distinguish the star-to-star abundance variations reported in Figure 1 from those due to stellar evolution effects. Figure 2 shows our sample in the $[\mathrm{N} / \mathrm{Fe}]-\log g$ plane. Stars from all GCs exhibit strong [N/Fe] differences at near constant $\log g$, which shows that stars at nearly the same evolutionary stage have vastly different $[\mathrm{N} / \mathrm{Fe}]$ abundances, which argues against evolutionary effects. We conclude that the intra-GC abundance variations reported in Figure 1 1 are indicative of the presence of multiple populations in our sample GCs.

It is the first time that the presence of spreads in the abundances of light elements has been established for the GCs in our sample, although for at least one of them (NGC 6528) it has been previously suggested by studies based on medium-resolution spectroscopy (Martell \& Smith 2009). Such data provide valuable constraints on model predictions in a regime where they have not been sufficiently tested. In particular, the presence of large star-to-star abundance variations - especially in the case of $\mathrm{Al}$ - for highmetallicity GCs challenge model predictions suggesting that 
Table 2. Stellar parameters, elemental abundances, radial velocities, and cluster-centric distances for sample stars. Errors reported in all quantities correspond to pipeline precision estimates. $T_{\text {eff }}$ and $\log g$ precision in DR12 is $\sim 90 \mathrm{~K}$ and $\sim 0.1$ dex.

\begin{tabular}{|c|c|c|c|c|c|c|c|c|c|c|}
\hline APOGEE ID & $T_{\text {eff }}$ & $\log g$ & {$[\mathrm{Fe} / \mathrm{H}]$} & {$[\mathrm{C} / \mathrm{Fe}]$} & {$[\mathrm{N} / \mathrm{Fe}]$} & {$[\mathrm{Na} / \mathrm{Fe}]$} & {$[\mathrm{Mg} / \mathrm{Fe}]$} & {$[\mathrm{Al} / \mathrm{Fe}]$} & $\mathrm{RV}(\mathrm{km} / \mathrm{s})$ & $\mathrm{d}\left({ }^{\prime}\right)$ \\
\hline $\begin{array}{l}\text { 2M17434071-2613528 } \\
\text { 2M17434331-2610217 } \\
2 \mathrm{M} 17434675-2616068\end{array}$ & $\begin{array}{l}3675 \\
3983 \\
4135 \\
\end{array}$ & $\begin{array}{l}0.1 \\
0.8 \\
1.2\end{array}$ & $\begin{aligned}-1.00 & \pm 0.04 \\
-0.85 & \pm 0.04 \\
-0.77 & \pm 0.04\end{aligned}$ & $\begin{array}{l}-0.02 \pm 0.05 \\
-0.10 \pm 0.06 \\
-0.10 \pm 0.06\end{array}$ & $\begin{array}{l}\text { Palomar } 6 \\
+0.18 \pm 0.09 \\
+0.08 \pm 0.09 \\
+0.69 \pm 0.10\end{array}$ & $\begin{array}{l}- \\
-\end{array}$ & $\begin{array}{l}+0.24 \pm 0.05 \\
+0.26 \pm 0.05 \\
+0.14 \pm 0.06\end{array}$ & $\begin{array}{l}-0.02 \pm 0.09 \\
+0.14 \pm 0.09 \\
+0.26 \pm 0.10\end{array}$ & $\begin{aligned} 178.621 & \pm 0.003 \\
175.579 & \pm 0.009 \\
175.28 & \pm 0.01\end{aligned}$ & $\begin{array}{l}0.63 \\
3.00 \\
2.94\end{array}$ \\
\hline $\begin{array}{l}2 \mathrm{M} 17475169-2443153 \\
2 \mathrm{M} 17480088-2447295 \\
2 \mathrm{M} 17480576-2445000 \\
2 \mathrm{M} 17480668-2447374 \\
2 \mathrm{M} 17481414-2446299\end{array}$ & $\begin{array}{l}3844 \\
3974 \\
3999 \\
3925 \\
3725\end{array}$ & $\begin{array}{l}1.4 \\
1.3 \\
1.1 \\
0.8 \\
0.9\end{array}$ & $\begin{aligned} &-0.31 \pm 0.03 \\
&-0.48 \pm 0.03 \\
&-0.61 \pm 0.04 \\
&-0.56 \pm 0.04 \\
&-0.02 \pm 0.03\end{aligned}$ & $\begin{array}{l}+0.15 \pm 0.04 \\
-0.34 \pm 0.05 \\
+0.02 \pm 0.05 \\
-0.39 \pm 0.05 \\
+0.09 \pm 0.04\end{array}$ & $\begin{array}{c}\text { Terzan } 5 \\
-0.14 \pm 0.07 \\
+1.11 \pm 0.07 \\
+0.77 \pm 0.09 \\
+1.07 \pm 0.08 \\
+0.18 \pm 0.06\end{array}$ & $\begin{array}{l}+0.15 \pm 0.12 \\
+0.47 \pm 0.11 \\
- \\
+0.25 \pm 0.12 \\
+0.05 \pm 0.09\end{array}$ & $\begin{array}{l}-0.22 \pm 0.04 \\
+0.04 \pm 0.04 \\
+0.21 \pm 0.06 \\
+0.12 \pm 0.04 \\
+0.04 \pm 0.04\end{array}$ & $\begin{array}{c}- \\
+0.62 \pm 0.06 \\
+0.16 \pm 0.09 \\
+0.37 \pm 0.07 \\
-0.09 \pm 0.05\end{array}$ & $\begin{aligned}-75.52 & \pm 0.02 \\
-99.471 & \pm 0.006 \\
-76.79 & \pm 0.02 \\
-89.922 & \pm 0.008 \\
-76.05 & \pm 0.01\end{aligned}$ & $\begin{array}{l}4.60 \\
1.17 \\
1.76 \\
0.96 \\
2.12\end{array}$ \\
\hline 2M18032356-3001588 & 4088 & 1.0 & $-1.09 \pm 0.04$ & $-0.38 \pm 0.07$ & $\begin{array}{c}\text { NGC } 6522 \\
+1.04 \pm 0.05\end{array}$ & - & $+0.17 \pm 0.06$ & $+0.40 \pm 0.11$ & $-13.76 \pm 0.01$ & 2.28 \\
\hline $\begin{array}{l}2 \mathrm{M} 18044775-3003469 \\
2 \mathrm{M} 18045107-3002378\end{array}$ & $\begin{array}{l}4167 \\
4168\end{array}$ & $\begin{array}{l}1.7 \\
1.7\end{array}$ & $\begin{array}{l}-0.16 \pm 0.04 \\
-0.21 \pm 0.04\end{array}$ & $\begin{array}{l}+0.18 \pm 0.05 \\
+0.03 \pm 0.05\end{array}$ & $\begin{array}{c}\text { NGC } 6528 \\
+0.22 \pm 0.07 \\
+0.76 \pm 0.08\end{array}$ & $\begin{array}{l}+0.23 \pm 0.12 \\
+0.61 \pm 0.12\end{array}$ & $\begin{array}{l}+0.16 \pm 0.05 \\
+0.14 \pm 0.05\end{array}$ & $\begin{array}{l}+0.19 \pm 0.07 \\
+0.36 \pm 0.07\end{array}$ & $\begin{array}{l}215.40 \pm 0.03 \\
210.75 \pm 0.02\end{array}$ & $\begin{array}{l}0.58 \\
0.81\end{array}$ \\
\hline $\begin{array}{l}\text { 2M18085726-2558403 } \\
\text { 2M18090968-2554574 } \\
\text { 2M18091335-2548357 } \\
\text { 2M18091466-2552275 } \\
\text { 2M18091564-2556008 } \\
\text { 2M18091666-2554424 } \\
\text { 2M18091912-2553326 } \\
\text { 2M18092147-2556039 } \\
\text { 2M18092234-2554381 } \\
\text { 2M18092241-2557595 } \\
\text { 2M18092826-2558233 } \\
\text { 2M18093498-2549038 }\end{array}$ & $\begin{array}{l}3762 \\
4817 \\
4566 \\
4153 \\
4057 \\
3899 \\
4409 \\
4126 \\
4428 \\
4145 \\
4440 \\
4830\end{array}$ & $\begin{array}{l}1.3 \\
2.4 \\
2.5 \\
1.6 \\
1.3 \\
1.1 \\
1.8 \\
1.3 \\
2.0 \\
1.3 \\
2.0 \\
2.6\end{array}$ & $\begin{aligned} &+0.20 \pm 0.03 \\
&-0.02 \pm 0.04 \\
&+0.08 \pm 0.03 \\
&-0.21 \pm 0.03 \\
&-0.18 \pm 0.03 \\
&-0.22 \pm 0.03 \\
&-0.08 \pm 0.04 \\
&-0.18 \pm 0.04 \\
&-0.23 \pm 0.04 \\
&-0.22 \pm 0.03 \\
&-0.10 \pm 0.04 \\
&-0.17 \pm 0.04\end{aligned}$ & $\begin{array}{l}+0.13 \pm 0.03 \\
-0.30 \pm 0.06 \\
+0.15 \pm 0.05 \\
-0.12 \pm 0.04 \\
-0.24 \pm 0.04 \\
-0.15 \pm 0.04 \\
-0.40 \pm 0.05 \\
-0.31 \pm 0.04 \\
+0.11 \pm 0.05 \\
+0.11 \pm 0.05 \\
-0.33 \pm 0.05 \\
+0.07 \pm 0.06\end{array}$ & $\begin{array}{l}\text { NGC } 6553 \\
-0.03 \pm 0.05 \\
+0.74 \pm 0.08 \\
+0.25 \pm 0.07 \\
+0.97 \pm 0.07 \\
+0.91 \pm 0.07 \\
+0.79 \pm 0.06 \\
+0.98 \pm 0.08 \\
+0.96 \pm 0.07 \\
+0.26 \pm 0.08 \\
+0.19 \pm 0.07 \\
+0.90 \pm 0.08 \\
+0.06 \pm 0.09\end{array}$ & $\begin{array}{l}- \\
+0.29 \pm 0.12 \\
+0.05 \pm 0.09 \\
+0.51 \pm 0.10 \\
+0.46 \pm 0.09 \\
+0.41 \pm 0.09 \\
+0.35 \pm 0.11 \\
+0.50 \pm 0.10 \\
+0.23 \pm 0.12 \\
+0.11 \pm 0.11 \\
+0.38 \pm 0.11 \\
-0.13 \pm 0.11\end{array}$ & $\begin{array}{l}+0.12 \pm 0.03 \\
-0.07 \pm 0.05 \\
+0.03 \pm 0.04 \\
+0.13 \pm 0.04 \\
+0.08 \pm 0.04 \\
+0.12 \pm 0.04 \\
+0.08 \pm 0.05 \\
+0.13 \pm 0.04 \\
+0.14 \pm 0.05 \\
+0.18 \pm 0.05 \\
+0.15 \pm 0.05 \\
+0.02 \pm 0.05\end{array}$ & $\begin{array}{c}+0.11 \pm 0.04 \\
- \\
-0.06 \pm 0.07 \\
+0.41 \pm 0.06 \\
+0.47 \pm 0.06 \\
+0.31 \pm 0.05 \\
+0.36 \pm 0.07 \\
+0.39 \pm 0.06 \\
+0.06 \pm 0.08 \\
+0.41 \pm 0.07 \\
+0.39 \pm 0.07 \\
+0.28 \pm 0.09\end{array}$ & $\begin{array}{c}9.571 \pm 0.009 \\
5.06 \pm 0.05 \\
-8.85 \pm 0.03 \\
2.41 \pm 0.01 \\
-1.02 \pm 0.02 \\
-11.115 \pm 0.004 \\
3.92 \pm 0.02 \\
7.01 \pm 0.01 \\
-0.80 \pm 0.02 \\
-7.94 \pm 0.01 \\
-1.02 \pm 0.02 \\
-15.65 \pm 0.02\end{array}$ & $\begin{array}{l}6.18 \\
1.84 \\
6.01 \\
2.17 \\
1.56 \\
0.29 \\
1.03 \\
1.77 \\
1.06 \\
3.63 \\
4.54 \\
6.71\end{array}$ \\
\hline
\end{tabular}

Table 3. Stellar parameters, elemental abundances, radial velocities, and angular cluster-centric distances for stars rejected on the basis of angular cluster-centric distances and/or radial velocities. Errors reported in all quantities correspond to pipeline precision estimates. $T_{\text {eff }}$ and $\log g$ precision in DR12 is $\sim 90 \mathrm{~K}$ and $\sim 0.1$ dex.

\begin{tabular}{|c|c|c|c|c|c|c|c|c|c|c|}
\hline APOGEE ID & $T_{\text {eff }}$ & $\log g$ & {$[\mathrm{Fe} / \mathrm{H}]$} & {$[\mathrm{C} / \mathrm{Fe}]$} & {$[\mathrm{N} / \mathrm{Fe}]$} & {$[\mathrm{Na} / \mathrm{Fe}]$} & {$[\mathrm{Mg} / \mathrm{Fe}]$} & {$[\mathrm{Al} / \mathrm{Fe}]$} & $\mathrm{RV}(\mathrm{km} / \mathrm{s})$ & $\mathrm{d}\left({ }^{\prime}\right)$ \\
\hline & & & & & Terzan 5 & & & & & \\
\hline 2M17480857-2446033 & 3687 & 0.2 & $-0.72 \pm 0.04$ & $+0.00 \pm 0.05$ & $+0.47 \pm 0.08$ & $-0.03 \pm 0.15$ & $+0.24 \pm 0.05$ & $+0.13 \pm 0.07$ & $-64.38 \pm 0.01$ & 1.09 \\
\hline 2M17483971-2452162 & 3938 & 1.0 & $-0.21 \pm 0.03$ & $+0.05 \pm 0.04$ & $+0.12 \pm 0.07$ & $+0.00 \pm 0.10$ & $+0.21 \pm 0.04$ & $+0.22 \pm 0.06$ & $-62.50 \pm 0.01$ & 9.64 \\
\hline 2M18053866-3008454 & 4346 & 1.8 & $-0.12 \pm 0.04$ & $-0.01 \pm 0.05$ & $\begin{array}{c}\text { NGC } 6528 \\
-0.07 \pm 0.08\end{array}$ & $-0.36 \pm 0.13$ & $+0.15 \pm 0.05$ & $-0.36 \pm 0.08$ & $+185.66 \pm 0.07$ & 11.88 \\
\hline & & & & & NGC 6553 & & & & & \\
\hline 2M18082912-2548259 & 3830 & 1.1 & $+0.06 \pm 0.03$ & $+0.01 \pm 0.03$ & $+0.34 \pm 0.05$ & $+0.32 \pm 0.07$ & $+0.00 \pm 0.03$ & $+0.07 \pm 0.04$ & $+20.062 \pm 0.002$ & 12.50 \\
\hline 2M18084319-2547042 & 3678 & 0.4 & $-0.37 \pm 0.03$ & $+0.20 \pm 0.04$ & $+0.26 \pm 0.07$ & $+0.24 \pm 0.11$ & $+0.24 \pm 0.04$ & $+0.18 \pm 0.06$ & $+24.817 \pm 0.005$ & 10.75 \\
\hline $2 \mathrm{M} 18084368-2557107$ & 4260 & 1.7 & $-0.09 \pm 0.03$ & $+0.14 \pm 0.05$ & $+0.15 \pm 0.07$ & $+0.03 \pm 0.11$ & $+0.26 \pm 0.05$ & $+0.21 \pm 0.07$ & $+3.22 \pm 0.02$ & 8.09 \\
\hline 2M18085792-2547267 & 3665 & 0.8 & $-0.22 \pm 0.03$ & $+0.27 \pm 0.04$ & $+0.25 \pm 0.06$ & $+0.31 \pm 0.11$ & $+0.20 \pm 0.04$ & $-0.23 \pm 0.06$ & $-9.56 \pm 0.01$ & 8.35 \\
\hline 2M18092650-2541199 & 3885 & 1.3 & $+0.07 \pm 0.03$ & $+0.05 \pm 0.04$ & $+0.26 \pm 0.05$ & $+0.22 \pm 0.07$ & $+0.02 \pm 0.03$ & $-0.05 \pm 0.04$ & $+17.440 \pm 0.005$ & 13.34 \\
\hline 2M18094548-2554255 & 4002 & 1.2 & $-0.24 \pm 0.03$ & $+0.15 \pm 0.04$ & $+0.21 \pm 0.07$ & $+0.19 \pm 0.12$ & $+0.20 \pm 0.04$ & $+0.19 \pm 0.07$ & $+19.44 \pm 0.01$ & 6.26 \\
\hline 2M18095105-2600134 & 3893 & 0.9 & $-0.29 \pm 0.03$ & $+0.16 \pm 0.04$ & $+0.17 \pm 0.07$ & $+0.13 \pm 0.12$ & $+0.30 \pm 0.04$ & $+0.54 \pm 0.06$ & $-30.917 \pm 0.009$ & 9.43 \\
\hline
\end{tabular}

the amplitude of star-to-star variations should decrease towards high metallicity (e.g., Karakas 2010; Ventura et al. 2013; Bastian et al. 2015; Di Criscienzo et al. 2016). A detailed confrontation between our data and model predictions is beyond the scope of this paper. In the remainder of this Section we briefly discuss the data for each GC separately.

\section{$3.1 \quad$ NGC 6553}

One of the most metal-rich GCs known in the Galaxy with $[\mathrm{Fe} / \mathrm{H}] \sim-0.2$ (e.g., Cohen et al. 1999; Origlia et al. 2002; Alves-Brito et al. 2006; Johnson et al. 2014; Tang et al. 2016), NGC 6553 is moderately massive $\left(3 \times 10^{5} M_{\odot}\right.$ McLaughlin \& van der Marel 2005), so one would expect it to host a measurable spread in the abundances of $\mathrm{Na}$ (Carretta et al. 2010) and N (Schiavon et al. 2013). It is also the $\mathrm{GC}$ for which our sample is the largest, so the finding 


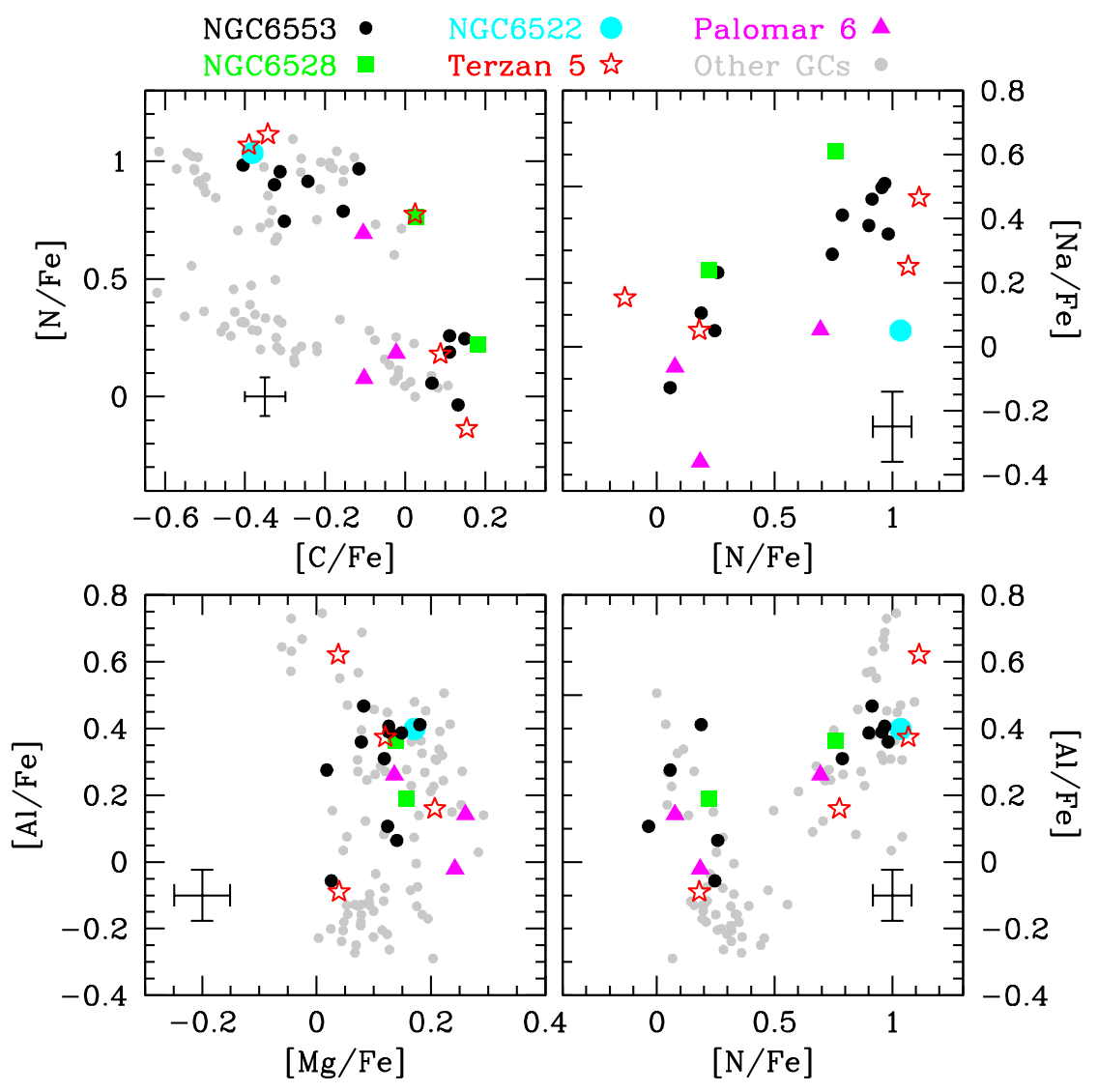

Figure 1. Stars from our GC sample in abundance space. Symbol types and colours are as indicated in the legend. "Other GCs" include DR12 data for halo/thick disk M 3, M 5, M 107, M 71, and NGC 6760 (Schiavon et al. 2016). See text for details.

of a large spread in the abundances of $\mathrm{N}(\sim 1$ dex $), \mathrm{Na}$ $(\sim 0.5 \mathrm{dex}), \mathrm{C}(\sim 0.5 \mathrm{dex})$, and $\mathrm{Al}(\sim 0.5 \mathrm{dex})$ is statistically robust. Interestingly, $\mathrm{Al}$ abundances in a couple of the N-normal stars are as high as those in their N-rich counterparts. Except for the presence of a few outliers that are probable non-members (see Tang et al. 2016), no clear spread in the abundances of heavy elements such as Fe or $\mathrm{Ca}$ is detected. The abundances of $\mathrm{N}, \mathrm{C}$, and perhaps $\mathrm{Na}$ seem to be bimodal, but any firm conclusion should await a substantial increase in sample size. In a separate publication (Tang et al. 2016), a careful evaluation of membership and a quantitative comparison between model predictions and observations is pursued for the case of NGC 6553, for which our stellar sample is the largest. We refer the reader to that paper for a more detailed analysis of the APOGEE data for that cluster.

\subsection{Terzan 5}

Terzan 5 is a peculiar object. Its colour-magnitude diagram displays two well-separated red horizontal branches (Ferraro et al. 2009), and it is known to host a very large population of millisecond pulsars (Ransom et al. 2005).
It is one of the few Galactic GCs for which a considerable spread in $[\mathrm{Fe} / \mathrm{H}]$ and possibly age has been detected (e.g., Ferraro et al. 2009, 2016). Indeed, Origlia et al. (2013) and Massari et al. (2014a b) used Keck/NIRSPEC, Keck/DEIMOS, and VLT/FLAMES spectra to establish the presence of at least three well defined stellar populations in Terzan 5 , with $[\mathrm{Fe} / \mathrm{H}] \sim-0.8,-0.3$, and +0.2 (the highest metallicity detected in any Galactic GC. Interestingly, Origlia et al. (2011) found that the relation between the abundances of $\mathrm{Fe}$ and the $\alpha$ elements $\mathrm{Mg}$ and $\mathrm{O}$ mimics that of the Galactic bulge itself. Unlike other massive Galactic GCs that show both a spread in Fe and lightelement abundances (for a review see Da Costa 2015), no spreads and anti-correlations between the abundances of light elements were previously detected in Terzan 5 (e.g., Origlia et al. 2011, 2013). This is surprising given its high mass $\left(\sim 2 \times 10^{6} M_{\odot} ;\right.$ Ferraro \& Lanzoni 2012) and the high metallicity of some of its stellar populations. These results led to the suggestion that Terzan 5 is the remnant core of a dwarf galaxy, or perhaps even an early fragment of the original bulge formation (Ferraro \& Lanzoni 2012).

Our sample for Terzan 5 consists of only five stars, yet the data reveal a large spread in light-element abundances, 


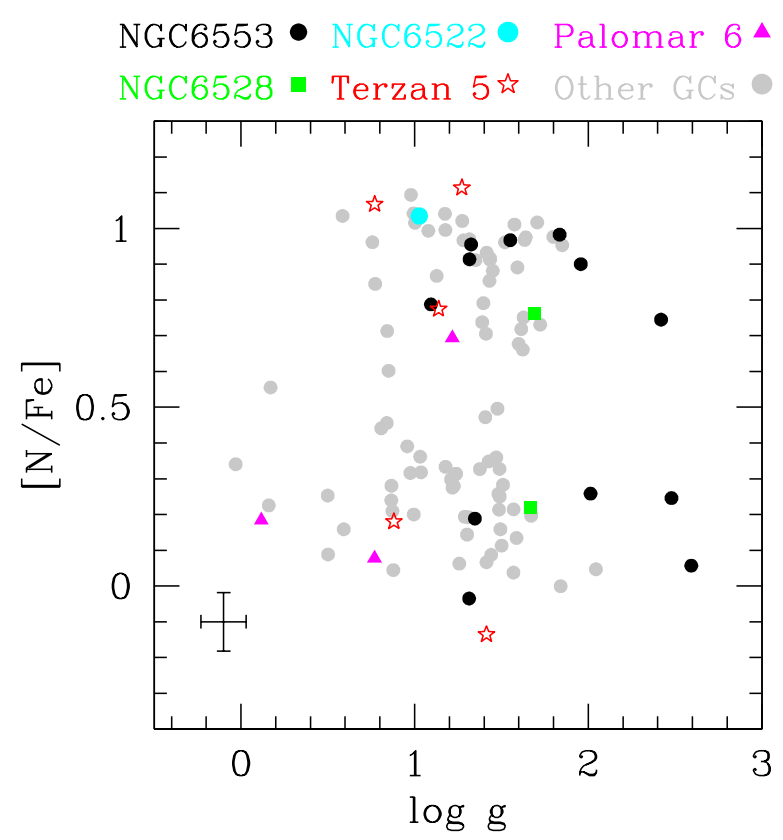

Figure 2. Nitrogen abundance ratios as a function of surface gravity for the sample stars. Stars of approximately the same gravity (thus same evolutionary stage) have vastly different $[\mathrm{N} / \mathrm{Fe}]$, which cannot be explained by mixing.

including a strong $\mathrm{N}-\mathrm{C}$ anti-correlation and equally strong $\mathrm{N}-\mathrm{Na}$ and $\mathrm{N}-\mathrm{Al}$ correlations. Membership uncertainties are obviously a concern in this case. All five stars fall safely within the metallicity range established by Massari et al. (2014b), although the N-rich sub-sample is particularly metal-poor, especially considering a zero-point correction of -0.2 dex to APOGEE raw $[\mathrm{Fe} / \mathrm{H}]$ (Holtzman et al. 2015). Origlia et al. (2013) measured the systemic radial velocity of Terzan 5 to be $-82 \mathrm{~km} / \mathrm{s}$, with a velocity dispersion of $\sigma \sim 15 \mathrm{~km} / \mathrm{s}$. All but one of our sample stars are situated within $1 \sigma$ of Terzan 5's systemic radial velocity. The exception is the N-rich star 2M17480088-2447295, with $v_{r}=-99.5 \mathrm{~km} / \mathrm{s}$ - which is too low by $\sim 2.5 \mathrm{~km} / \mathrm{s}$ to be within $1 \sigma$ from the GC systemic RV. Since this star has a very high $[\mathrm{N} / \mathrm{Fe}]$ and, at a distance of $\sim 1^{\prime}$ from the cluster centre, is well within its tidal radius $\left(\sim 6 .^{\prime} 7\right)$, we deem it a very likely member of Terzan 5 .

ASPCAP results for some of the Terzan 5 candidate members merit a more detailed look. Some of the elemental abundances for star 2M17475169-2443153 are quite unusual, with $[\mathrm{N} / \mathrm{Fe}]=-0.14,[\mathrm{Mg} / \mathrm{Fe}]=-0.22$ and $[\mathrm{Al} / \mathrm{Fe}]=-1$. Visual inspection showed that there is an important mismatch between synthetic and observed spectra at the continuum level through a large fraction of the APOGEE spectral interval. In those regions, the normalized flux in continuum pixels of the observed spectrum is higher than unity and higher than their counterparts in the synthetic spectrum. This mismatch is probably due to a problem in the normalization of the observed spectrum. Such a mismatch is most likely responsible for an underestimate of the $\mathrm{N}, \mathrm{Na}$, and Mg abundances, as lines due to CN (e.g., $\lambda \lambda 15322,15332 \AA$ ) and $\mathrm{Mg}$ (e.g., $\lambda \lambda 15339,15959 \AA$ ) are affected by this issue particularly strongly. Interestingly, an acceptable match of the observed spectrum by ASPCAP is found in regions of known $\mathrm{OH}$ (e.g., $\lambda \lambda 16708,16719,16889 \AA$ ) and $\mathrm{CO}$ (e.g., $\lambda \lambda 15368,15997,16189 \AA$ ) lines (García Pérez et al. 2016). Perhaps most importantly, such a systematic effect on the continuum normalization could also potentially lead to an overestimate of surface gravity, which may impact the values of abundances inferred from molecular lines which are sensitive to $\log g$. Indeed, the ASPCAP surface gravity for this $\operatorname{star}(\log g=1.4)$ is quite high for such a cool star $\left(T_{\text {eff }}=3844 \mathrm{~K}\right)$, which could be the result of the continuum systematics identified above. Without further calculations it is impossible to gauge the impact of this effect on the chemical composition of this star. Given the good fit to CO and $\mathrm{OH}$ lines, the abundance of $\mathrm{C}$ is probably reliable, modulo $\log g$ effects. On the other hand, we consider the abundances of $\mathrm{N}, \mathrm{Mg}$, and $\mathrm{Na}$ to be questionable.

Regarding $\mathrm{Al}$, the situation is more complex, as ASPCAP reports $[\mathrm{Al} / \mathrm{Fe}]=-1$ for $2 \mathrm{M} 17475169-2443153$, which we find suspiciously low. The strengths of all three $\mathrm{Al}$ lines in the APOGEE spectrum $(\lambda \lambda 16723,16755$, and $16767 \AA)$ are overpredicted by ASPCAP, suggesting that $[\mathrm{Al} / \mathrm{Fe}]$ for this star should be even lower. However, comparison of the spectrum of 2M17475169-2443153 with those of stars with very similar stellar parameters and CNO abundances (e.g., 2M06182536+3414581, 2M17493226-2309585, 2M18322950-1246417, and 2M18493324-0302028) but vastly different $[\mathrm{Al} / \mathrm{Fe}]$ (ranging from -0.1 to -0.59 ) showed very similar line strengths, which is surprising. We do not understand the origin of these issues, so we choose not to consider the abundances of $\mathrm{Mg}, \mathrm{Na}$ and $\mathrm{Al}$ for this star.

We also examined the abundances of star 2M17480576-2445000, particularly due to the fact that ASPCAP found a very low value for $[\mathrm{Na} / \mathrm{Fe}]=-0.47$, which is surprising due to its very large $[\mathrm{N} / \mathrm{Fe}]=+0.77$. Inspection of the two $\mathrm{Na}$ lines in the APOGEE region suggests that $[\mathrm{Na} / \mathrm{Fe}]$ for this star is not reliable. The strongest of the two lines $(\lambda 16393 \AA)$ is lost to bad pixels, whereas the remaining weaker line $(\lambda 16378 \AA)$ is severly underestimated by ASPCAP, despite the fact that the observed continuum is well matched by the best fitting synthetic spectrum. On the other hand, ASPCAP does an excellent job of matching the $\mathrm{CN}$ lines in the spectrum of this star, so that we consider that $[\mathrm{N} / \mathrm{Fe}]$ is quite reliable. We therefore decide to ignore the abundance of $\mathrm{Na}$ for this star in the remainder of our analysis.

We conclude that there is strong evidence for the presence of an intrinsic spread, as well as correlations and anticorrelations between light-element abundances in Terzan 5 . A more detailed study, based on a larger sample and including additional elemental abundances is in order. 


\subsection{NGC 6528}

With $[\mathrm{Fe} / \mathrm{H}] \sim-0.2$, NGC 6528 is another very metalrich bulge GC (Carretta et al. 2001; Barbuy et al. 2004; Zoccali et al. 2004; Origlia et al. 2005). It is also moderately massive $\left(\sim 2 \times 10^{5} M_{\odot} ;\right.$ McLaughlin \& van der Marel 2005). Based on medium-resolution spectroscopy of a relatively small sample, Martell \& Smith (2009) determined the presence of a bimodal distribution of $\mathrm{CN}$ band strengths. In contrast, Calamida et al. (2014) obtained Strömgren photometry for a larger sample, but did not report the presence of a bimodal distribution in CN-sensitive colour indices. Our sample contains only two NGC 6528 candidate members, with essentially identical atmospheric parameters, but a significantly different $\mathrm{N}$ and $\mathrm{C}$, and $\mathrm{Na}$ abundances suggesting the presence of an abundance spread in this GC as well, in agreement with the result by Martell \& Smith (2009).

\subsection{Palomar 6}

Lee et al. (2004) determined the metallicity of Palomar 6 to be $[\mathrm{Fe} / \mathrm{H}] \sim-1$. The cluster is moderately massive ( $\sim$ $2.3 \times 10^{5} M_{\odot} ;$ Boyles et al. 2011), and therefore we would expect in this case to detect the presence of multiple populations. The sample of candidate members is small, and the only element found to present variations that are statistically significant is $\mathrm{N}$. While there are indications that $\mathrm{C}$ and $\mathrm{Al}$ also show variations, they are not significantly larger than the error bars. The data on $\mathrm{Na}$, on the other hand, suggest the presence of variations. However, at the relatively low metallicity of Palomar 6, Na abundances in DR12 are uncertain, due to the weakness of the available lines in the APOGEE spectrum. We conclude that a definite variation in $[\mathrm{N} / \mathrm{Fe}]$ was detected, but for $\mathrm{C}, \mathrm{Al}$, and $\mathrm{Na}$ more data are required to establish the definitive presence of abundance spreads.

\section{$3.5 \quad$ NGC 6522}

An old GC located in the inner Galaxy, NGC 6522 is moderately metal poor with $[\mathrm{Fe} / \mathrm{H}] \sim-1$ (Barbuv et al. 2009) and has a low mass $\left(\sim 6 \times 10^{4} M_{\odot}\right.$, Gnedin \& Ostriker 1997). It is thus not immediately clear that NGC 6522 is expected to contain multiple populations. Our sample contains only one candidate member of this GC, which makes any conclusion on the presence of multiple populations by definition very uncertain. However, the star is likely a member on the grounds of metallicity, radial velocity, and position, and it has a very high nitrogen abundance $([\mathrm{N} / \mathrm{Fe}]=+1.04)$. Moreover, in Figure 2 one can see that our NGC 6522 sample star occupies the same locus in [N/Fe]-log $g$ space as second generation stars in other GCs. Indeed, at the metallicity of NGC 6522, only SG stars attain such high nitrogen abundances in the APOGEE DR12 data (Schiavon et al. 2016, Figure 1). Therefore, we conclude that NGC 6522 is likely to host multiple populations, but a strong statement to that effect must await the availability of good quality data for a larger sample.

\section{CONCLUSIONS}

As part of the SDSS-III/APOGEE survey, we have obtained elemental abundances for 23 candidate members of five massive globular clusters located in the inner Galaxy, including some of the most metal-rich known Galactic GCs. Our main conclusions are summarised as follows:

- Spreads in $[\mathrm{N} / \mathrm{Fe}]$ have been detected in all GCs for which more than one star was observed, and all these abundances could be measured. Among the latter GCs, all but Palomar 6 also exhibit significant spreads in [C/Fe], [Al/Fe], and $[\mathrm{Na} / \mathrm{Fe}]$. The standard anti-correlation between $\mathrm{N}$ and $\mathrm{C}$, and correlations between $\mathrm{N}$ and $\mathrm{Al}$ and $\mathrm{Na}$ are also present in our data. This result indicates the prevalence of the multiple-population phenomenon in GCs as metal-rich as $[\mathrm{Fe} / \mathrm{H}] \sim-0.1$. In at least one $\mathrm{GC}$ for which our sample is largest (NGC 6553), there is strong evidence for a bimodal distribution in the abundances of $\mathrm{C}$ and $\mathrm{N}$. We conclude that $\mathrm{N}$-rich stars are present in metal-rich GCs, and therefore they can be used as reliable tracers of field GC-like populations over a wide range of metallicities. As a corollary, the absence of a large population of metal-rich N-rich stars in the sample discovered by Schiavon et al. (2016) in the inner Galaxy reflects the metallicity distribution function of the presumptive destroyed GCs;

- The presence of large spreads in $[\mathrm{N} / \mathrm{Fe}]$ in metal-rich GCs is interesting also from the point of view of stellarevolution models, as predictions from different groups seem to imply a strong dependence on metallicity of the yields of certain elements (e.g., Karakas 2010; Ventura et al. 2013; Di Criscienzo et al. 2016). A large spread of $\mathrm{Al}$ abundances in GCs of near-solar metallicity may constitute an important challenge to existing models. It would be naive to rule out a metallicity dependence of stellar yields on the basis of our results alone, but hopefully our numbers can be used for careful comparison with detailed model predictions based on state-of-the-art yields;

- For most of the GCs in our study the presence of multiple populations has been uncovered for the first time. This is the case for NGC 6553 (see also Tang et al. 2016), NGC 6528, and Palomar 6. In the case of NGC 6522, the sample contains only one star, which happens to have a $\mathrm{SG}$ abundance pattern. Since no GC is known to host only SG stars, it is very likely that NGC 6522 also contains multiple populations. For Terzan 5, although multiple populations were known to be present with different $[\mathrm{Fe} / \mathrm{H}]$, it is the first time that star-to-star variations in light elements is reported;

- The case of Terzan 5 is particularly interesting, as it is a very massive $\mathrm{GC}$ with a substantial spread in $[\mathrm{Fe} / \mathrm{H}]-\mathrm{a}$ feature that places it in the same category as objects like $\omega$ Cen, M54, and others, which are often referred to as remnants of dwarf satellites accreted to the Galaxy (for a short review, see Da Costa 2015). It is interesting, in this regard, that Terzan 5 displays abundance spreads and correlations similar to those found in $\omega$ Cen (Johnson \& Pilachowski 2010), which suggests that these systems may occupy a 
region of parameter space somewhere in-between globular clusters and dwarf spheroidal galaxies.

The pursuit of a solution to the enigma of globular cluster formation and its connections with the formation of the Galaxy itself will receive a great boost once a massive database of chemistry and kinematics of members of all Galactic GCs is in place. Despite valiant efforts, we are still scratching the surface. Further progress is expected from observations taken from the Southern hemisphere with APOGEE-2 (e.g., Maiewski et al. 2016). In addition, the advent of high-resolution NIR spectrographs with large multiplexing power and small fiber-collision radii, such as MOONS (Cirasuolo et al. 2014), will potentially bring about a paradigm shift in this field.

\section{ACKNOWLEDGMENTS}

R.P.S. thanks Maurizio Salaris and Nate Bastian for useful comments on an early version of the manuscript. The anonymous referee is thanked for a careful reading of the manuscript and valuable suggestions. R.E.C. acknowledges support from Gemini-CONICYT for Project 32140007". S.L.M. acknowledges support from Australian Research Council grant DE140100598. Sz.M. has been supported by the Premium Postdoctoral Research Program of the Hungarian Academy of Sciences, and by the Hungarian NKFI Grants K-119517 of the Hungarian National Research, Development and Innovation Office. D.G., and B.T. gratefully acknowledge support from the Chilean BASAL Centro de Excelencia en Astrofísica y Tecnologías Afines (CATA) grant PFB-06/2007. S.R.M. thanks NSF grant AST-1312863 T.C.B. acknowledges partial support for this work from grants PHY 08-22648; Physics Frontier Center/Joint Institute for Nuclear Astrophysics (JINA), and PHY-1430152; Physics Frontier Center/JINA Center for the Evolution of the Elements (JINA-CEE), awarded by the US National Science Foundation. D.A.G.H. was funded by the Ramón y Cajal fellowship number RYC-2013-14182 and he acknowledges support provided by the Spanish Ministry of Economy and Competitiveness (MINECO) under grant AYA-2014-58082P.

Funding for SDSS-III has been provided by the Alfred P. Sloan Foundation, the Participating Institutions, the National Science Foundation, and the U.S. Department of Energy Office of Science. The SDSS-III web site is http://www.sdss3.org/. SDSS-III is managed by the Astrophysical Research Consortium for the Participating Institutions of the SDSS-III Collaboration including the University of Arizona, the Brazilian Participation Group, Brookhaven National Laboratory, University of Cambridge, Carnegie Mellon University, University of Florida, the French Participation Group, the German Participation Group, Harvard University, the Instituto de Astrofísica de Canarias, the Michigan State/Notre Dame/JINA Participation Group, Johns Hopkins University, Lawrence Berkeley National Laboratory, Max Planck Institute for Astrophysics, New Mexico State University, New York Univer- sity, Ohio State University, Pennsylvania State University, University of Portsmouth, Princeton University, the Spanish Participation Group, University of Tokyo, University of Utah, Vanderbilt University, University of Virginia, University of Washington, and Yale University.

\section{REFERENCES}

Alam, S., Albareti, F. D., Allende Prieto, C., et al. 2015, ApJS, 219, 12

Alves-Brito, A., Barbuy, B., Zoccali, M. et al. 2006, A\&A, 460, 269

Barbuy, B., Meléndez, J., Ortolani, S., et al. 2004, Mem. Soc. Astron. Italiana, 75, 398

Barbuy, B., Zoccali, M., Ortolani, S., et al. 2009, A\&A, 507, 405

Bastian, N., Lamers, H. J. G. L. M., de Mink, S. E., et al. 2013, MNRAS, 436, 2398

Bastian, N., Cabrera-Ziri, I., \& Salaris, M. 2015, MNRAS, 449, 3333

Bica, E., Clariá, J. J., Piatti, A. E., \& Bonatto, C. 1998, A\&AS, 131, 483

Boyles, J., Lorimer, D. R., Turk, P. J., et al. 2011, ApJ, 742,51

Calamida, A., Bono, G., Lagioia, E. P., et al. 2014, A\&A, 565, A 8

Carretta, E., Cohen, J. G., Gratton, R. G., \& Behr, B. B. 2001, AJ, 122, 1469

Carretta, E., Bragaglia, A., Gratton, R. G., et al. 2010, A\&A, 516, A55

Cirasuolo, M., Afonso, J., Carollo, M., et al. 2014, SPIE, 9147, 91470N

Cohen, J. G., Gratton, R. G., Behr, B. B., \& Carretta, E. 1999, ApJ, 523, 739

Da Costa, G. S. 2015, arXiv:1510.00873

Di Criscienzo, M., Ventura, P., Garcia-Hernandez, D. A., et al. 2016, MNRAS, in press, arXiv:1607.02876

Eisenstein, D. J., Weinberg, D. H., Agol, E., et al. 2011, AJ, 142,72

Ferraro, F. R., Dalessandro, E., Mucciarelli, A. et al. 2009, Natur, 462, 483

Ferraro, F. R., \& Lanzoni, B. 2012, Memorie della Societa Astronomica Italiana Supplementi, 19, 129

Ferraro, F. R., Massari, D., Dalessandro, E., et al. 2016, ApJ, 828, 75

García Pérez A.E. et al. 2016a, AJ, 151, 144

Gnedin, O. Y. \& Ostriker, J. P. 1997, ApJ, 474, 223

Gratton, R., Sneden, C., \& Carretta, E. 2004, ARA\&A, 42, 385

Gunn, J. E., Siegmund, W. A., Mannery, E. J., et al. 2006, AJ, 131, 2332

Harris, W. E. 1996, AJ, 112, 1487

Harris, W. E. 2010, arXiv:1012.3224

Holtzman, J. A., Shetrone, M., Johnson, J. A., et al. 2015, AJ, 150,148

Hopkins, P. F. 2014, ApJ, 797, 59

Johnson, C. I., \& Pilachowski, C. A. 2010, ApJ, 722, 1373 
Johnson, C. I., Rich, R. M., Kobayashi, C., Kunder, A. \& Koch, A. 2014, AJ, 148, 67

Karakas, A. I. 2010, MNRAS, 403, 1413

Larsen, S. S., Brodie, J. P., Forbes, D. A., \& Strader, J. 2014, A\&A, 565, A98

Lee, J.-W., Carney, B. W. \& Balachandran, S. C. 2004, AJ, 128,2388

Majewski, S.M., Schiavon, R. P., Frinchaboy, P. et al. 2016, AJ, submitted, arXiv:1509.05420

Martell, S. L., \& Smith, G. H. 2009, PASP, 121, 577

Massari, D., Mucciarelli, A., Ferraro, F. R. et al. 2014a, ApJ, 795, 22

Massari, D., Mucciarelli, A., Ferraro, F. R. et al. 2014b, ApJ, 791, 101

McLaughlin, D. E. \& van der Marel, R. P. 2005, ApJS, 161, 304

Mészáros, Sz., Allende Prieto, C., Edvardsson, B., et al. 2012, AJ, 144, 120

Mészáros, Sz., S., Martell, S. L., Shetrone, M., et al. 2015, AJ, 149, 153

Nidever, D. L., Holtzman, J. A., Allende Prieto, C., et al. 2015, AJ, 150, 173

Origlia, L., Rich, R. M., \& Castro, S. 2002, AJ, 123, 1559

Origlia, L., Valenti, E., \& Rich, R. M. 2005, MNRAS, 356, 1276

Origlia, L., Rich, R. M., Ferraro, F. R. et al. 2011, ApJL, 726, L20

Origlia, L., Massari, D., Rich, R. M., et al. 2013, ApJL, 779 , L5

Ortolani, S., Bica, E., \& Barbuy, B. 1997, A\&AS, 126,

Pancino, E., Rejkuba, M., Zoccali, M. \& Carrera, R. 2010, A\&A, 524, A44

Piotto, G. 2008, MmSAI, 79, 334

Piotto, G., King, I. R., Djorgovski, S. G., et al. 2002, A\&A, 391, 945

Piotto, G., Milone, A. P., Bedin, L. R., et al. 2015, AJ, 149, 91

Pipino, A., Chiappini, C., Graves, G., \& Matteucci, F. 2009, MNRAS, 396, 1151

Ransom, S. M., Hessels, J. W. T., Stairs, I. H., et al. 2005, Science, 307, 892

Renzini, A. 2008, MNRAS, 391, 354

Renzini, A., D'Antona, F., Cassisi, S., et al. 2015, MNRAS, 454, 4197

Sakari, C., Shetrone, M., Schiavon, R. P., et al. 2016, ApJ, in press.

Schiavon, R. P., Caldwell, N., Conroy, C., et al. 2013, ApJ, 776, L7

Schiavon, R. P., Zamora, O., Carrera, R., et al. 2016, MNRAS, in press, arXiv:1606.05651

Shetrone, M., Bizyaev, D., Lawler, J. E., et al. 2015, ApJS, 221,24

Skrutskie, M. F., Cutri, R. M., Stiening, R., et al. 2006, AJ, 131, 1163

Tang, B., Cohen, R., Geisler, D. 2016, ApJ, in press, arXiv:1610.06763

Valenti, E., Ferraro, F. R., \& Origlia, L. 2010, MNRAS, 402, 1729
Ventura, P., Di Criscienzo, M., Carini, R., \& D'Antona, F. 2013, MNRAS, 431, 3642

Wilson, J. C., Hearty, F., Skrutskie, M. F., et al. 2012, Proc. SPIE, 8446, 84460

Zamora, O., García-Hernández, D. A., Allende Prieto, C., et al. 2015, AJ, 149, 181

Zasowski, G., Johnson, J. A., Frinchaboy, P. M., et al. 2013, AJ, 146, 81

Zoccali, M., Barbuy, B., Hill, V., et al. 2004, A\&A, 423, 507

(C) 2016 RAS, MNRAS 000, 1010 